(C) 1996 IEEE. Personal use of this material is permitted. Permission from IEEE must be obtained for all other uses, in any current or future media, including reprinting/republishing this material for advertising or promotional purposes, creating new collective works, for resale or redistribution to servers or lists, or reuse of any copyrighted component of this work in other works. 


\title{
APPLYING UNDERSAMPLING TO THE NUCLEAR MAGNETIC RESONANCE SIGNAL
}

\author{
Pablo Pérez, Juan José Vaquero, Andrés Santos, Manuel Rivera, Francisco del Pozo \\ Grupo de Bioingeniería y Telemedicina, E.T.S.I. Telecomunicación (U.P.M.) \\ 28040 Madrid, Spain. \\ E-mail: andres@teb.upm.es
}

\begin{abstract}
This paper presents the use of undersampling in an NMR prototype equipment. The results show that undersampling is a convenient tool to be used in the processing of these signals. It allows to easily transform bandpass Free Induction Decay (FID) signals, centered at high frequencies, into lowpass signals or bandpass signals at much lower frequencies. Main advantages of using this technique are improved signal to noise ratio and analog electronic stages suppression.
\end{abstract}

\section{INTRODUCTION}

In a typical Nuclear Magnetic Resonance imaging system, the signal obtained in the receiver coil is amplified using tuned amplifiers, processed with demodulators and intermediate frequency amplifiers (IF), and finally by a quadrature phase sensitive detector. The last step produces two FID (Free Induction Decay) signals, which must be filtered to remove high-frequency components [1]. Among other problems of this classic architecture, the analog mixer required is a component that generates a relatively large noise power [2].

These analog sections can be replaced by using digital techniques, which reduce the noise level and the phase distortion, and therefore increase the quality of the final images. One of these digital techniques is oversampling, but then the high conversion rate needed implies the use of fast electronic circuits and large storage capacities. Another alternative is the use of undersampling techniques or passband sampling, which have been successfully applied in the radiocommunications field, in what is called software radio. In software radio an analog to digital converter takes the IF signal and samples it at a rate below the Nyquist frequency. This sampling process causes the replication of the IF signal spectrum at the baseband, i.e. undersampling moves the spectrum to a lower frequency, functioning like a mixer [3]. The original information could be completely recovered from these sampled values if we ensure that the signal has no frequency components above a frequency $f_{h}$ or below a frequency $f_{i}$ and $\left(f_{h}-f_{l}\right) \leq f_{1}$. Passband signal must also satisfy $[4,5]$

$$
\frac{2 f_{h}}{n} \leq f_{x} \leq \frac{2 f_{1}}{(n-1)}
$$

where $n$ is an integer that fulfills

$$
2 \leq n \leq \frac{f_{h}}{\left(f_{h}-f_{1}\right)}
$$

Basically, the NMR signal is a passband signal with a center frequency which can take values from a few megahertz to a few hundred megahertz, depending on the main magnetic field used. In this work we show the use of bandpass sampling in the processing of signals coming from an NMR equipment and the advantages of this method.

Undersampling directly applied to the amplified resonance signal allows to reduce the speed and storage requirements, and therefore the costs of implementation. The sample and hold circuit is the only component that must keep the same characteristics when oversampling or undersampling is used, with the purpose that it could manage all the frequency components contained in the original signal.

\section{METHOD}

In order to evaluate if undersampling is a viable alternative to detect and process the NMR signal, we have performed some tests using a prototype of nuclear magnetic resonance equipment, which operates at $5.017 \mathrm{MHz}$. This equipment was completely developed with analog circuitry. During this evaluation the NMR signals were sampled, quantized and processed using a digital oscilloscope (Tektronix TDS-524A with GPIB interface). This oscilloscope can manage up to $500 \mathrm{MH} z$ signals and different sampling rates can be used. It allows to acquire the signals with undersampling and oversampling techniques.

The FID signal has been sampled at two different points of the NMR system:

- After the tuned filters that receive the signal from the coil: in this point the signal is a passband signal and has a carrier frequency equal to the Larmor frequency $(5.017$ $\mathrm{MHz}$ )

- The equipment output, where the signal is in baseband after the detection, filtering and amplifying stages.

Signals in both points were acquired with a sampling rate of $50 \mathrm{Ksamples} / \mathrm{s}$ and their spectra were obtained.

\section{RESULTS}

As shown in Fig. 1, the resonance information is completely recovered in a lower frequency, just by undersampling according to the specified conditions. The 
signal carrier, sampled at $50 \mathrm{Ksamples} / \mathrm{s}$, has a frequency of $17 \mathrm{kHz}$, equal to the difference between the Larmor freguency and the closest lower multiple of the sampling rate. There are two important points to remark in this experiment:

- The FID signal spectrum should not be shifted to baseband because that would produce spectrum overlap (the sampling rate needed for this shift does not fulfill equations (1) and (2)).

- The passband FID signal can be converted to lowpass by using the adequate sampling rate, that in this case is a frequency equal to $24.95 \mathrm{kHz}$ for $2.5 \mathrm{kHz}$ bandwidth. This value was not used in the experiments shown here because the oscilloscope cannot select this sampling rate.

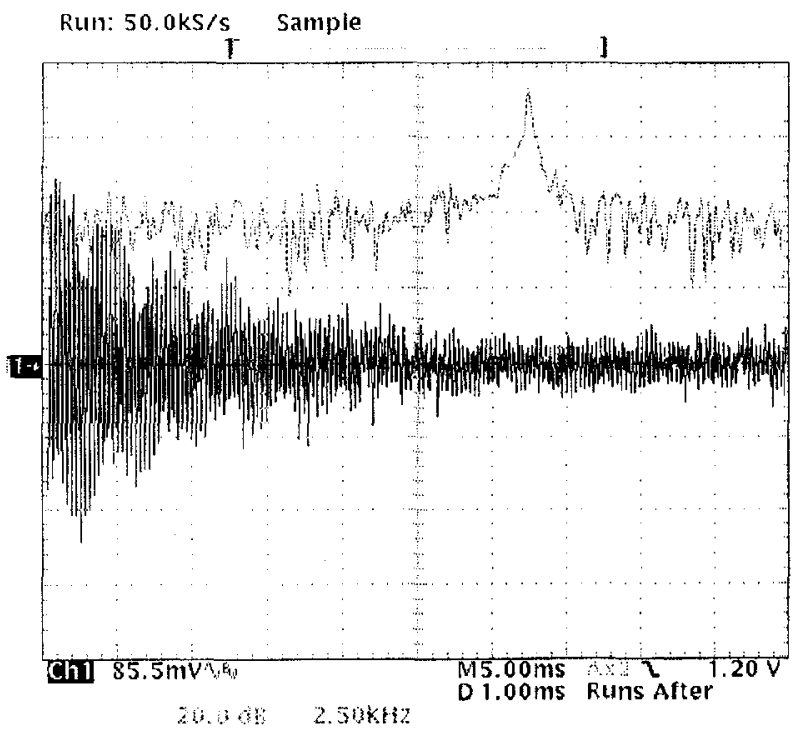

Fig. 1. The undersampled FID signal is shown in the center with its spectrum above. For the spectrum, the vertical divisions are $20 \mathrm{~dB}$ and the horizontal ones, $2.5 \mathrm{kHz}$. For the undersampled FID signal these values are $85.5 \mathrm{mV}$ and $5 \mathrm{~ms}$. Note the peak in the spectrum at $17 \mathrm{kH} /$.

Fig. 2 presents the resonant FID signal in baseband as it is obtained after its analog processing: detection, filtering and amplification. Signal shown in Fig. 2 has been amplified with tuned amplificrs which introduce a noticeable phase distortion.

We have repeated the process using different sampling rates $(100), 250$ y $500 \mathrm{Ksamples} / \mathrm{s})$ with the same results.

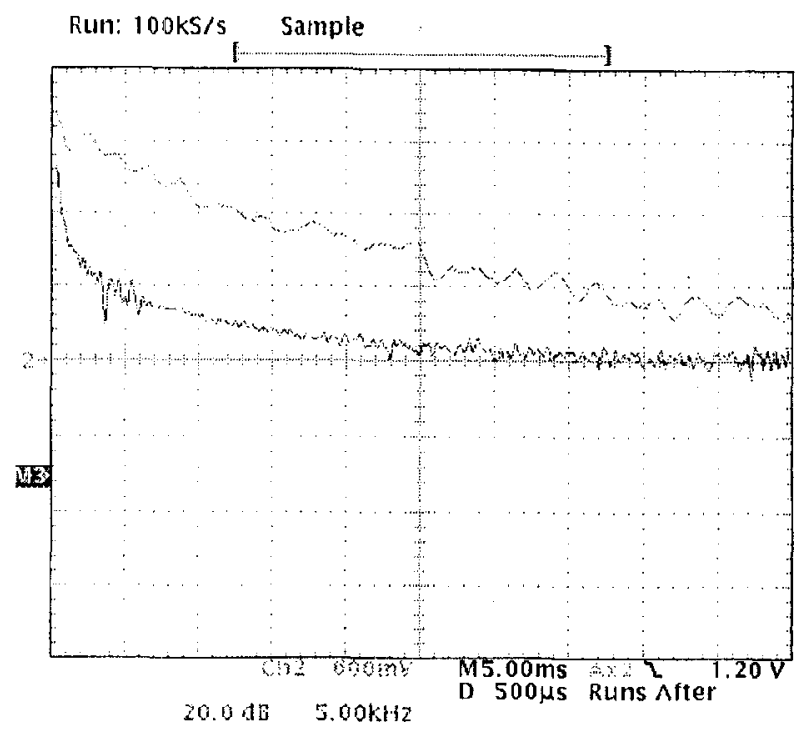

Fig. 2. Real part of the FID signal in baseband (above) and its spectrum (midalle line). Vertical and horizontal scalc divisions for the spectrum are $20 \mathrm{~dB}$ and $2.5 \mathrm{kHz}$ respectively. These values for the FID signal are $600 \mathrm{mV}$ and $5 \mathrm{~ms}$.

\section{CONCLUSIONS}

This work presents undersampling as an alternative way of processing NMR signals. Our experimental results demonstrate that this technique can be very useful for NMR signal processing. This alternative procedure eliminates some analog electronic circuits, replacing them with low speed digital processing (almost baseband or audio band). The specd and storage requirements are then reduced in comparison with systems working with the Nyquist criteria: a sampling frequency of $50 \mathrm{kHz}$ is used instead of $10 \mathrm{MHz}$. The resulting signals have a SNR higher than those obtained with a much more complex analog electronics. Further digital processing (at this low speed) could certainly improve the characteristics of the NMR signal.

\section{REFERENCES}

11] C-N. Chen and D. I. Hoult, Biomedical magnetic resonance technology. Adam Hilger, 1989.

[2] H. Taub and D. I. Schilling, Principles of communication systems. McGraw Hill Kogakusha, 1971.

[3] B. Schweber, "Converters restructure communication architectures," EDN, August 3, 1995, pp. 51-64.

[4] E. O. Brigham, The Fast Fourier Transform and its applications. Prentice Hall, 1988.

[5] J. A. Wepman, "Analog-to-digital converters and their applications in radio receivers," IEEE Communications Magazine. pp. 39-45, May 1995. 